\title{
Single Fluorescent Molecule Confocal Microscopy: A New Tool for Molecular Biology Research and Biosensor Development
}

C. Darrow, T. Huser, C. Campos, M. Yan, S. Lane, R. Balhorn

February 11, 2000

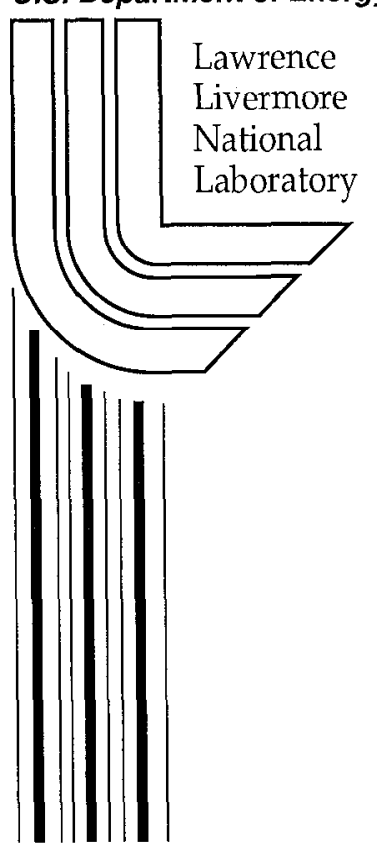




\section{DISCLAIMER}

This document was prepared as an account of work sponsored by an agency of the United States Government. Neither the United States Government nor the University of California nor any of their employees, makes any warranty, express or implied, or assumes any legal liability or responsibility for the accuracy, completeness, or usefulness of any information, apparatus, product, or process disclosed, or represents that its use would not infringe privately owned rights. Reference herein to any specific commercial product, process, or service by trade name, trademark, manufacturer, or otherwise, does not necessarily constitute or imply its endorsement, recommendation, or favoring by the United States Government or the University of California. The views and opinions of authors expressed herein do not necessarily state or reflect those of the United States Government or the University of California, and shall not be used for advertising or product endorsement purposes.

Work performed under the auspices of the U. S. Department of Energy by the University of California Lawrence Livermore National Laboratory under Contract W-7405-Eng-48.

This report has been reproduced directly from the best available copy.

Available to DOE and DOE contractors from the

Office of Scientific and Technical Information

P.O. Box 62, Oak Ridge, TN 37831

Prices available from (423) 576-8401

http://apollo.osti.gov/bridge/

Available to the public from the National Technical Information Service

U.S. Department of Commerce 5285 Port Royal Rd., Springfield, VA 22161 hltp://www.ntis.gov/

OR

Lawrence Livermore National Laboratory Technical Information Department's Digital Library http://www.llnl.gov/tid/Library.html 


\title{
FY99 Midyear LDRD Final Report
}

\author{
Single Fluorescent Molecule Confocal Microscopy: A New Tool for Molecular \\ Biology Research and Biosensor Development
}

Tracking Code: 99-ERD-067

\section{Introduction}

Our original proposal was presented to the LDRD committee on February 18 , 1999. The revised proposal that followed incorporated changes that addressed the issues, concerns, and suggestions put forth by the committee members both during the presentation and in subsequent discussions we've had with individual committee members. The goal of the proposal was to establish an SMD confocal microscopy capability and tcchnology basc at LLNL. Here we report on our progress during the 6month period for which funding was available.

We began by making modifications and upgrades to existing LLNL equipment. As was pointed out in our proposal, single-fluorescent-molecule confocal microscopy systems are not commercially available. The microscopy system we proposed was designed around a Zeiss Axiovert S100TV inverted microscope. The basic body of such a microscope was available at LLNL for our use. The output of a Coherent Inc. Argon ${ }^{+}$ laser $(488,514 \mathrm{~nm})$ was passed through an optical system that allowed the intensity of the beam to be properly adjusted by filtering prior to injection into a fiber optic. Fiber coupling to the microscope input optics is advantageous because in greatly increase the mechanical stability thereby reducing time spent on daily realignment. Light exiting the fiber was collimated by a complimentary objective (mounted on the microscope platform) and passed into the epi-fluorescence input port of the microscope. Fluorescence collected from the sample volume was passed vertically down through the microscope body to an optical system located below. This system consisted of the confocal aperture $(100 \mu \mathrm{m})$, band-pass filters, relay mirrors and optics, and an avalanche photo-diode detector (active-area diameter: $0.18 \mathrm{~mm}$ ). Due to its extreme sensitivity, the system was enclosed in a light-tight box that provided for fine adjustments of the detector position from outside the box. A schematic representation of the basic system is shown in Fig. 1.

The sample is placed at the focal plane of the objective where both excitation and emission collection occur. The samples are prepared on ultra-clean glass cover slips (processed with dilute HF and ultra-pure distilled water). Dilute sample (typically $10^{-11}$ molar) is applied to the slide using a spin-coater. Typically, only one or two samples are prepared at a time due to the fact that it is difficult to keep them contaminant free for extended periods of time. Presently our system is configured for scanned imaging. That is, images are built up pixel by pixel by recording the intensity of fluorescence at each position as the sample is rastered (i.e. translated in a grid-like pattern) in the focal plane over the region of interest. To accomplish this, the microscope was retrofitted with a sub- 
nanometer precision, active-feedback 3-axis piezoelectric scanning stage. A computerinterfaced controller provides scanning instructions to the piezo translation stage and records the intensity (as counts per second) from the avalanche photodiodes. The scanning stage allows regions up to $100 \mu \mathrm{m} \times 100 \mu \mathrm{m}$ in size to be investigated. Typically, we operate with 128 pixels per scan line over a line length of $5-20 \mu \mathrm{m}$. An image from one of LLNL's first SMD demonstration experiments is shown in Fig. 2 (a dry sample of rhodamine 6G on glass). An image of the microscope, as it appears in the lab, is shown in Fig. 2.

One fundamental question is, how one can be certain that the "dots" in the image of Fig. 3 are indeed from single molecules, and not from clumps of aggregated fluorescent molecules? This is a valid question since thousands of aggregated rhodamine molecules would be indistinguishable from single molecules in such a 2-D image. This is because the microscope cannot truly resolve either; both would appear as single points of light with a diameter corresponding to the diffraction-limited point-spread of the microscope optics, not the true diameter of the fluorescent source of light. This question is addressed by the insets of Fig. 3. First, if one observes the fluorescent emission of a single molecule as a function of time, an abrupt decrease of the fluorescence to background levels is eventually observed, often during one of the lines of the scan of that particular molecule. This is the well-known photo-physical effect of photo-bleaching by which an excited fluorescent molecule undergoes an irreversible reaction (often with oxygen) that renders it non-fluorescent. Such an abrupt quenching would not be possible for an aggregation of molecules since the likelihood that many molecules would simultaneously quench is negligible. Second it is possible for a temporary quenching of a single molecule to take place (see inset Fig. 3). In such events, a molecule temporarily stops emitting, but then a short time later begins to emit again, and continues to do so until complete quenching occurs. As is the case for simple quenching, this could not be observed if the point source were not a single molecule.

We have begun to consider other types of samples and applications. Applications are being considered that take advantage of our ability, through SMD, to detect single molecules (or very small numbers of single molecules). One such application is an assay for botulinum toxin (bot tox). Bot tox is increasingly a problem in agricultural consumer products (e.g. milk) because current techniques lack the sensitivity required to detect the toxin in livestock animals before it appears in potentially dangerous levels in food products. Also, there is increasing interest in developing methods for detecting the presence of the toxin in the biosecurity arena. We plan to investigate SMD for a bot tox assay by labeling at least two different toxin anti-bodies with different fluorescent labels. By adding a second detection channel to our SMD system, we will be able to simultaneously monitor both antibodies. Co-localization of the two antibodies within an SMD image will be evidence that the two were drawn together by the toxin. Thus, spatially coincident feature at the two wavelengths would be a positive indication for presence of the toxin since accidental co-localization can be made vanishingly small. The bot tox assay concept will form the basis for a request for renewal of FY00 Midyear LDRD funding for this project. 


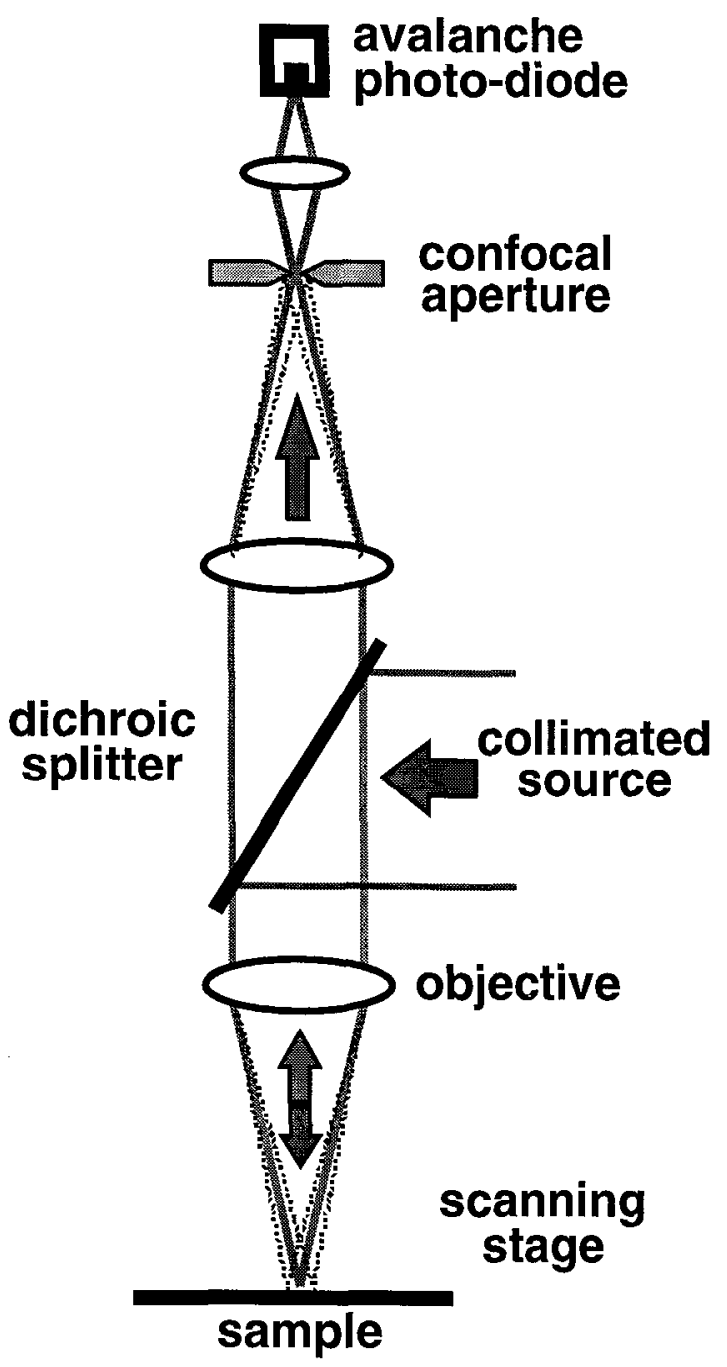

Fig. 1: Schematic Representation of the Confocal Single Molecule Detection Microscope. In the LLNL system, the collimated source is derived from argon-laser light emerging from a fiber optic. The dichroic splitter allows the passage of fluorescence while rejecting excitation light. The confocal (pinhole) aperture rejects any light that does not originate from the diffraction-limited focal spot of the microscope objective. 


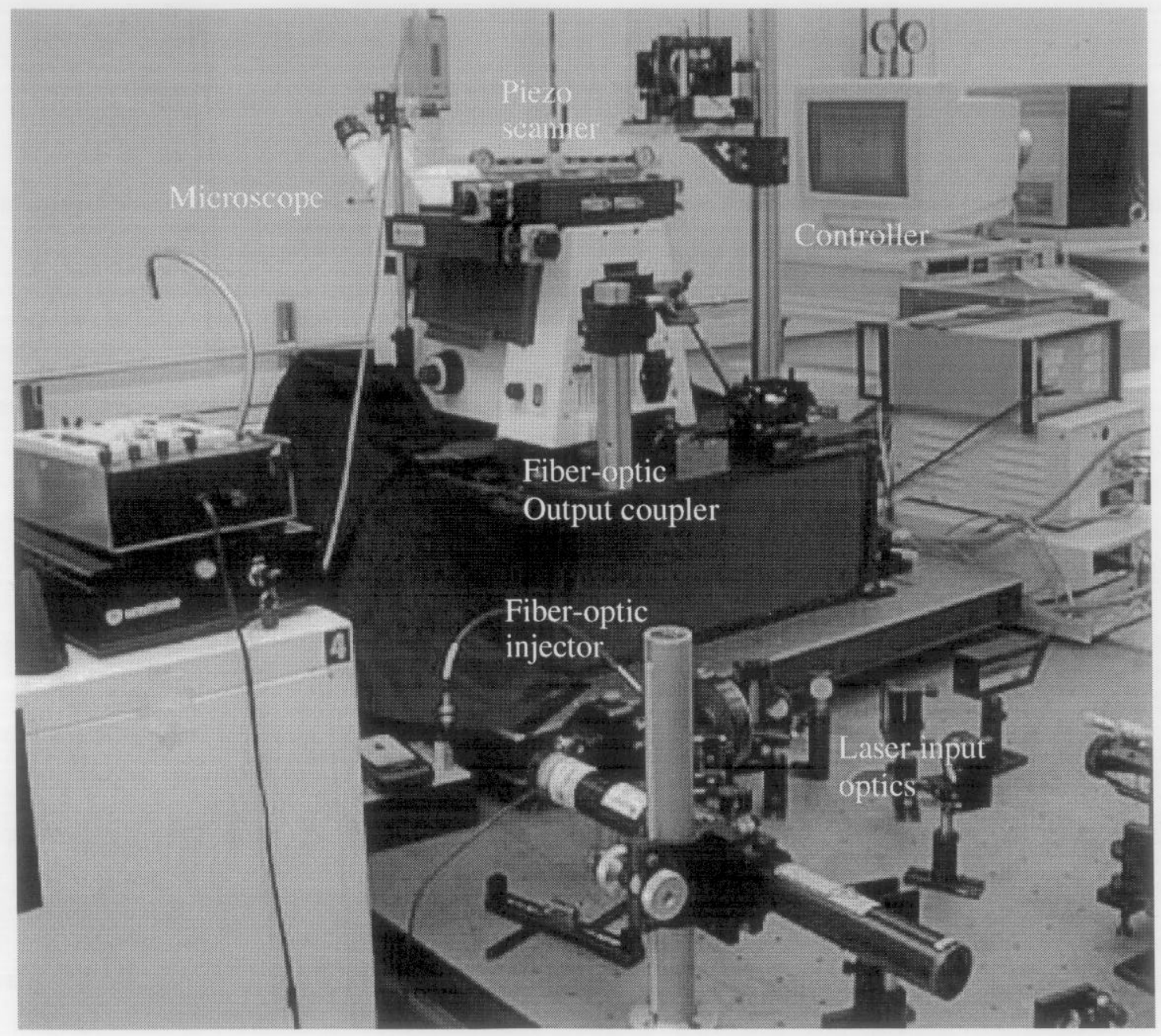

Fig. 2: Photograph depicting the confocal SMD microscope showing, the microscope, piezo scanner, computer controller/display, fiber optic input and output couplers, and laser alignment optics. No visible is the argon laser (to the right in the foreground) 

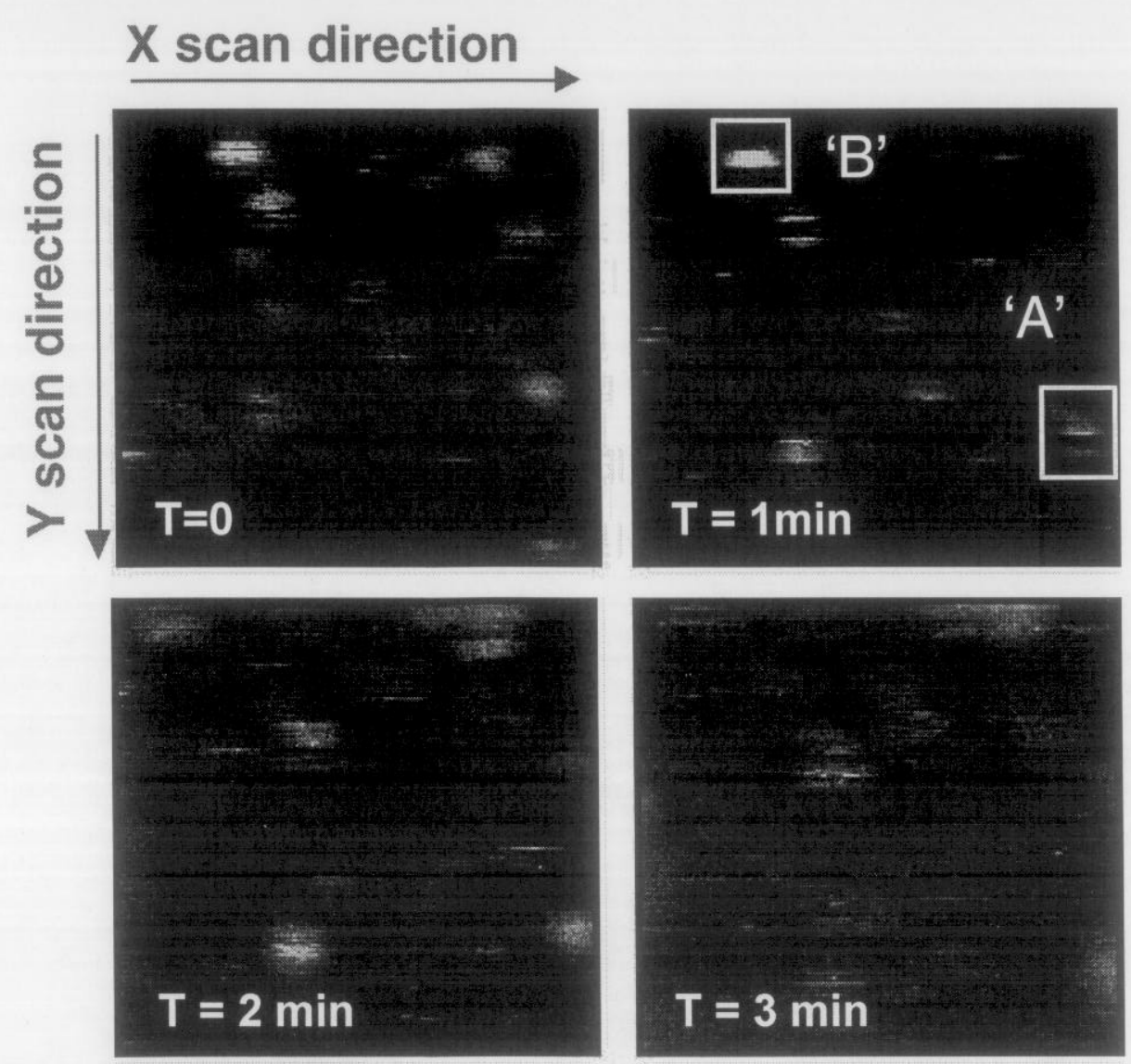

$\mathrm{T}=\mathbf{2} \mathrm{min}$

\section{$\mathrm{T}=3 \mathrm{~min}$}

\section{$5 \mu \mathrm{m}$}

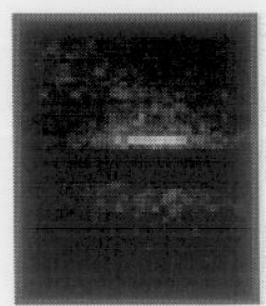

'A' temporary dark state

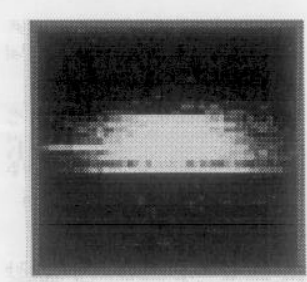

'B' photobleaching

Fig. 3: Example of SMD images from one of first SMD demonstration experiments. Sample is rhodamine 6-G. Four temporally sequential images are shown. Note progressive photobleaching of the molecules in the image. Insets ' $\mathrm{A}$ " and ' $\mathrm{B}$ ' correspond to two features in the " $\mathrm{T}=1 \mathrm{~min}$ " image; 'A' shows abrupt transition of a molecule to a temporary dark state; ' $\mathrm{B}$ ' shows abrupt photobleaching of a single molecule. Both are indicative of the fact that single molecules, not aggregates of many molecuels, are being detected. 